\title{
La densidad urbana como variable de análisis de la ciudad. El caso de Cuenca, Ecuador
}

\author{
M. Augusta Hermida. Universidad de Cuenca, Cuenca, Ecuador. \\ Carla Hermida. Universidad de Cuenca, Cuenca, Ecuador. \\ Natasha Cabrera. Universidad de Cuenca, Cuenca, Ecuador. \\ Christian Calle. Universidad de Cuenca, Cuenca, Ecuador.
}

RESUMEN | El debate teórico sobre la ciudad dispersa versus la ciudad compacta, en el mundo y particularmente en América Latina, pone en la palestra los aspectos clave necesarios para proponer un nuevo modelo de ciudad, donde la calidad de vida de las presentes y futuras generaciones sea el punto focal de reflexión. A través del estudio del estado del arte sobre el tema se concluye que el regreso a la ciudad compacta es la alternativa para un posible desarrollo sustentable en las ciudades intermedias de América Latina. En el caso de Cuenca, se concentra el estudio en las densidades de la ciudad desde los ańos cincuenta hasta nuestros días. Sorprende comprobar que Cuenca fue una ciudad compacta con alta calidad de vida pero que, paulatinamente, se está expandiendo innecesariamente, con todos los problemas que ello implica.

PALABRAS CLAVE | crecimiento urbano, sustentabilidad urbana, ciudades intermedias.

ABSTRACT |The theoretical debate about the scattered city versus the compact city, in the worldwide and particularly in Latin America, this put in evidence the necessary key aspects to propose a new city model, where the quality of life in the present and future generations will be the focus point of reflection. Throughout the study of the state of the art about this topic, it concludes that the returning to a compact city model is the alternative for a possible sustainable development in the intermediate cities of Latin America. In the Cuenca case is concentrated in the study of the densities of the city from the 1950s until today. It is amazing to prove that Cuenca was a compact city with a high quality of life, but gradually it is unnecessarily expanding, with all the problems involved.

KEYWORDS | urban growth, urban sustainability, intermediate cities.

Recibido el 26 de octubre de 2013, aprobado el 11 de julio de 2014

E-mail: Augusta Hermida, augusta.hermida@ucuenca.edu.ec| Carla Hermida, cmhermida@uc.cl | Natasha Cabrera, natasha.cabrera@ ucuenca.edu.ec | Christian Calle, cristian.calle@ucuenca.edu.ec 


\section{Agradecimientos}

Los autores expresan su agradecimiento a la Universidad de Cuenca, en particular a su Dirección de Investigación, por el financiamiento del proyecto "Modelos de Densificación Territorial para las zonas consolidadas de la ciudad de Cuenca", del cual forma parte este trabajo; al Departamento Interdisciplinario de Investigación en Espacio y Población y al Grupo Ciudades Sustentables-Llactalab, por su permanente apoyo. Igualmente agradecen de manera especial a los investigadores Pablo León y Jaime Tenezaca; a los ayudantes de investigación y digitadores que participaron en el proyecto: Carolina Abril Moscoso, María Emilia Durán Carrasco, María Laura Guerrero Balarezo, Sebastián Vanegas Bravo y Daniela Auquilla Clavijo; y a los estudiantes de la Facultad de Arquitectura que colaboraron en el levantamiento y digitalización de la información.

\section{Introducción}

La ciudad es uno de los productos más extraordinarios que ha creado la humanidad. Por definición es multifuncional, concentra la diversidad, y está en permanente construcción-reconstrucción, porque nunca se acaba; se trata de un "producto social" en proceso de cambio constante que tiene historia. (Carrión, 2001, p. 7)

El estudio de la densificación de las ciudades constituye una importante herramienta para evitar el crecimiento innecesario de la ciudad, aplicando para ello un enfoque de sustentabilidad que atienda varios frentes: proximidad, conectividad, población y actividad, transporte alternativo, habitabilidad del espacio público, complejidad urbana, dotación de espacios verdes y biodiversidad, autosuficiencia energética, autosuficiencia hídrica, adaptación y mitigación al cambio climático, cohesión social, dotación de equipamientos, etcétera. La densidad es uno de los componentes del debate teórico sobre la ciudad dispersa versus la ciudad compacta en el mundo y, particularmente, en América Latina.

El Ecuador, al igual que gran parte de los países latinoamericanos, experimentó a lo largo de la segunda mitad del siglo xx diversos fenómenos que determinaron la forma urbana, el crecimiento y las densidades habitacionales de las ciudades. Cuenca no fue ajena a las transformaciones urbanas resultantes de esos procesos, que incluyen -entre otros- el cambio de modelo económico hacia la industrialización sustitutiva de importaciones, que provocó una fuerte migración poblacional del campo a la ciudad; y, en el último cuarto del siglo anterior, el boom petrolero y la influencia del modelo neoliberal.

El artículo analiza la variación en las densidades brutas en el área urbana de Cuenca a partir de 1950, con la finalidad de establecer conclusiones y recomendaciones para definir un necesario nuevo modelo de ciudad, proponer otro enfoque a la planificación y abrir nuevas preguntas de investigación. Sorprende comprobar que Cuenca fue una ciudad compacta con alta calidad de vida, pero 
que, paulatinamente, se está convirtiendo innecesariamente en una ciudad dispersa, con todos los problemas que esto conlleva. Se concluye que el regreso a la ciudad compacta es la alternativa para un posible desarrollo sustentable en las ciudades intermedias de América Latina.

\section{El regreso a la ciudad compacta como alternativa para el desarrollo sustentable}

A pesar de las variantes acordes a cada país o periodo, se conoce como ciudad dispersa aquel modelo urbano de baja densidad que presenta discontinuidad en sus partes, con poca diversidad, baja proximidad de usos y que ocupa las periferias, en su mayoría, únicamente para vivienda unifamiliar, alejada de los centros urbanos y, por ende, de los servicios básicos y equipamientos (Lehmann, 2010; Navarro, 2011; Muñiz, Calatayud \& García, 2010; Arbury, 2005). Varias fueron las causas que contribuyeron a la dispersión de las ciudades; entre ellas, la necesidad de trasladarse hacia las periferias como reacción en contra de las malas condiciones de vida, contaminación y ruido de los centros urbanos (Muñiz et al., 2010; Rufí \& Pinatella, 2007); el avance en las tecnologías de la información y las comunicaciones (TIC), que disminuyeron el peso del factor distancia (Ministerio de Medio Ambiente de Espańa, 2007); la adquisición de vehículos privados que, para 1970, se habían convertido en componentes intrínsecos de la cotidianidad; la adquisición de viviendas en las periferias, que, de acuerdo con De Mattos (2010), se fundamentaba en el incremento del ingreso personal medio, la preferencia por una vivienda unifamiliar con jardín y la generación de grupos familiares más pequeños. A todo lo anterior se puede agregar la incorporación de la mujer al mercado laboral, lo cual modifica la manera como las personas viven, trabajan y viajan.

La ciudad dispersa trae consigo numerosos impactos económicos, sociales y ambientales. Como impactos económicos están aquellos relacionados, por un lado, con la provisión de servicios básicos, infraestructura y equipamientos que demandan las zonas alejadas de los centros urbanos; y, por otro, con los altos costos para el control de la contaminación atmosférica y la seguridad ante el tráfico ocasionado por el uso masivo del automóvil (Arbury, 2005). Los impactos sociales son los más difíciles de medir, pero son evidentes: inequidad; riesgos en la salud, ya que la ciudad dispersa desalienta la caminata y otras actividades físicas; pérdida de sentido de comunidad, segregación, polarización, reclusión residencial; pérdida de espacio público y desigualdad en el acceso a la movilidad, ya que se favorece principalmente al vehículo privado (Arbury, 2005; De Mattos, 2010; Muñiz et al., 2010). Duany, Plater-Zyberk y Speck (2000) (citado en Arbury, 2005) sostienen que la dispersión genera modelos de segregación; los niños que crecen en este tipo de asentamientos no desarrollan un sentido de comunidad y empatía con sus vecinos o habitantes de otros barrios, percibiéndolos con desconfianza e, inclusive, desprecio. Finalmente, en cuanto a impactos ambientales, se destaca la pérdida de suelo natural, causada por su uso excesivo en las periferias, que deriva en disminución de biodiversidad, distorsión del ciclo hídrico, afectación a los valores paisajísticos y contaminación del agua y del suelo (Ministerio de Medio Ambiente de España, 2007; Cervero, 1998); 
y en contaminación atmosférica y de ruido, debido al uso masivo del automóvil y el consumo excesivo de recursos no renovables para abastecer las necesidades de su uso (Muñiz et al., 2010). Nozzi (2003) (citado en Arbury, 2005) concluye que los modelos de ciudad dispersa causan un 20 a $50 \%$ más de contaminación que los modelos de ciudad compacta. A pesar de que los avances tecnológicos generan estrategias para reducir los impactos ambientales relacionados con la contaminación y el uso de recursos no renovables, no existe tecnología posible que contrarreste los impactos sociales que provoca un modelo de ciudad disperso (Cervero, 1998).

A partir de los ańos noventa, sin embargo, se inicia una nueva etapa, por lo menos en términos teóricos: la conciencia de la necesidad de un desarrollo sustentable, de la necesidad de salvaguardar los recursos naturales y de los impactos negativos de un modelo de desarrollo disperso, lo que lleva las miradas a la ciudad construida. A pesar de que desde los años setenta algunos autores promulgan la necesidad de un cambio de pensamiento sobre la planificación urbana, fue en los noventa que esta reflexión se intensifica. Según Dimitriou (2011), fue en 1987, con el Informe Brundtland Our Common Future (United Nations, 1987), donde se marca la necesidad de un cambio en la manera de producir ciudad. La Agenda 21, suscrita en la Cumbre de Río en 1992; la Carta del Nuevo Urbanismo, resultado del Primer Congreso del Nuevo Urbanismo en Estados Unidos, de 1993; la Carta de Alborg, a partir de la Conferencia sobre Ciudades y Poblaciones Sostenibles en 1994, son tan solo algunos de los ejemplos de compromisos y reflexiones sobre el nuevo enfoque que debe adoptar la planificación. Surgen así numerosas aproximaciones para abordar el tema de la ciudad, que apuntan a cambiar la tendencia del crecimiento desmedido para beneficio de las generaciones actuales y futuras. Entre tales aproximaciones están el smartgrowth, el new urbanism, el transit-oriented-development (TOD), el LEED-ND, el Active Living by Design (Ewing, Meakins, Bjarnson \& Hilton, 2011), el urbanismo verde o el urbanismo ecológico. De manera general, estos movimientos proponen cosas similares: la necesidad de incrementar la densidad, la diversidad, la accesibilidad, el transporte público y la cohesión social.

A pesar de que la mayoría de autores revisados considera que el modelo de la ciudad compacta es una mejor alternativa frente a las ciudades dispersas, aún existe una minoría que piensa lo contrario. Estos últimos aluden a que las ciudades dispersas y orientadas al automóvil son más agradables y ofrecen mayor confort para los ciudadanos. Según señala Gakeneheimer (2011), los que sostienen este punto de vista se basan en que la opción de ciudad compacta es poco deseada y poco factible, y en que algunas investigaciones demuestran que, pese a haberse incrementado drásticamente el número de vehículos y la cantidad de millas viajadas, las emisiones vehiculares se han reducido. De igual manera, Cervero (1998) destaca encuestas que demuestran una relación inversa entre la densidad residencial y el nivel de satisfacción con la calidad de vida: las personas prefieren su vivienda unifamiliar aislada. Este autor concluye que al elegir autoridades que pregonan los modelos de ciudad dispersa, la ciudadanía demuestra su preferencia individual por este tipo de desarrollo. En otras palabras, la brecha está entonces entre los intereses individuales versus los intereses colectivos. 
Una de las principales críticas al modelo de ciudad compacta radica en la paradoja según la cual para conseguir sustentabilidad, la ciudad debe ser más densa, pero para que una ciudad sea habitable, las funciones y la población deberían estar más dispersas (Neuman, 2005). Autores como Barton, Melia y Parkhurst (2011) señalan como uno de los principales factores que provocan el bajo nivel de habitabilidad en las ciudades densas, el incremento de los volúmenes de tráfico de manera local. Los volúmenes de tráfico locales son directamente proporcionales a la densidad poblacional, relación a la que estos autores llaman la "paradoja de la intensificación"; en otras palabras, se reduce el uso del vehículo per cápita, pero se incrementa la concentración vehicular en el ámbito local y, por ende, las emisiones. Por ello, para que la ciudad compacta sea sostenible deben existir varias medidas interrelacionadas (redes de tranvía, ciclovías, áreas peatonizadas, reducción de los límites de velocidad, restricción de estacionamiento, entre otras) que deberán sumarse a la densificación.

Moliní y Salgado (2010) sostienen que promover la ciudad compacta debe ser un recurso manejado con prudencia y flexibilidad; es decir, que las ciudades deben ser razonablemente compactas. Verdaguer (2011) considera que si bien las alternativas para solucionar los problemas de la ciudad difusa han sido positivas, no son directamente aplicables a las grandes extensiones urbanas. Las grandes ciudades ya nunca podrán ser compactas, pero esto no debe significar que no sean densas en sus asentamientos, ya que una adecuada densidad permitirá una distribución eficiente de los servicios (Herce \& Magrinyá, 2010).

A pesar de este debate inconcluso, la Agencia de Ecología Urbana de Barcelona ha realizado investigaciones en el tema, y en función de ello afirma que "tras el análisis comparado de diversos sistemas urbanos, el modelo urbano que mejor se ajusta al principio de eficiencia urbana y habitabilidad urbana es la ciudad compacta en su morfología, compleja en su organización, eficiente metabólicamente y cohesionada socialmente" (Rueda, 2011, p. 243). En el caso espańol, la base para la práctica del urbanismo ecológico la constituyen el Tomo i y el Tomo in del Libro Verde del Medio Ambiente Urbano, editado por el Ministerio de Medio Ambiente y la Agencia de Ecología Urbana de Barcelona (2007 y 2009). La Agencia considera que los procesos de transformación de las ciudades implican un análisis transversal de varias dimensiones que apuntan a mejorar la situación actual y dar respuestas adecuadas al futuro. Estas dimensiones se clasifican en cuatro ejes: compacidad, complejidad, eficiencia y cohesión social. Cada uno de ellos contiene a su vez dimensiones que pueden ser medibles, analizables, comparables y, sobre todo, son ámbitos en los cuales se puede intervenir para mejorar las condiciones de la ciudad.

Las dimensiones mencionadas difieren según cada lugar específico, pero de manera general se puede indicar que la compacidad se refiere a la realidad física del territorio y, por lo tanto, a la morfología urbana; de allí que aborde la densidad, la ocupación del suelo, el espacio público, la habitabilidad, la movilidad y los servicios. La complejidad, por su parte, se refiere a la organización urbana, a cómo se interrelacionan las actividades económicas, los equipamientos, las instituciones, y aborda indicadores tales como la mezcla de usos, los espacios verdes y la biodiversidad. La eficiencia tiene que ver con los flujos tanto materiales como de agua y energía; estos flujos soportan a los sistemas urbanos, y para su análisis se utilizan 
indicadores de metabolismo urbano. Finalmente, la cohesión social se refiere a las personas y sus relaciones, cómo se reúnen, quién ocupa los espacios, cómo realizan intercambios, etcétera. En este sentido, según Rueda (2008), "la ocupación dispersa genera patrones de vida poco sostenibles, mientras que una densidad adecuada, que no caiga en la congestión, permite conseguir una masa crítica de personas y actividades en cada entidad residencial, lo cual permite la dotación de transporte público, los servicios y equipamientos básicos y las dotaciones comerciales imprescindibles para desarrollar la vida cotidiana desde patrones de proximidad" (p. 18).

Por otra parte, y según Borsdorf (2003), al concentrar la mirada en América Latina se puede observar que la evolución urbana de sus ciudades, posterior a la conquista española, está marcada por cuatro fases: la ciudad colonial, sectorial, polarizada y finalmente fragmentada. Las dos primeras se caracterizan por ser compactas, y se mantienen hasta aproximadamente la década de 1920. A partir de esa fecha y hasta 1970, se experimenta una ciudad polarizada: la forma urbana se determina por la industrialización que trajo consigo la migración campo-ciudad; es en esas décadas que se intensifica el contraste entre ciudad rica y pobre. En la periferia se ubican, por un lado, los barrios exclusivos de la clase alta y, por otro, las zonas de vivienda social e informal. Este fenómeno de urbanización espontánea y de autoconstrucción, que en su mayoría se refiere a ocupaciones ilegales (Ludeña, 2006), caracteriza a algunas ciudades latinoamericanas. De acuerdo con Carrión (2001), es en esas décadas que las ciudades latinoamericanas inician "una nueva lógica de urbanización (...) basada en la periferización y la metropolización, propias del modelo económico de la sustitución de importaciones y del Estado de Bienestar" (p. 7).

A partir de los setenta, la mayoría de los países latinoamericanos "cambiaron a un nuevo paradigma económico, basado en el neoliberalismo (...) La retirada del Estado, la privatización y la desregulación permitieron a los inversores, planificadores y ciudadanos, mayores libertades" (Borsdorf, 2003, p. 37). Consecuentemente, las ciudades se expanden, utilizando suelos anteriormente rurales y agrícolas. Claras muestras de dispersión en Latinoamérica constituyen las ciudades de México D.F., Santiago, Buenos Aires, São Paulo, Medellín, Bogotá, entre otras. De acuerdo con De Mattos (2010), esta dinámica, en Latinoamérica, se observa no solo en las grandes metrópolis, sino también en urbes de menor dimensión, como Cali, Concepción, Córdoba, Guadalajara, Montevideo, San José de Costa Rica y Quito. Sostiene, además, que a pesar de las diferencias de implantación del modelo en cada ciudad latinoamericana, este ha evolucionado bajo las mismas directrices que en otras partes del mundo.

Posteriormente, en los noventa, las ciudades latinoamericanas se ven inmersas en importantes procesos de liberalización, globalización, descentralización y democratización (Comisión Económica para América Latina y el Caribe [CEPAL], 1998). Carrión (2001) señala que en este contexto de globalización, las ciudades latinoamericanas también inician un proceso de reflexión teórica y vuelven la mirada a la ciudad construida. De acuerdo con el autor, son tres los procesos concurrentes que determinan este giro: los cambios demográficos, que -al contrario de años anteriores- a partir de finales del siglo xx tienden a que la tasa de migración del campo a la ciudad sea cada vez menor; "la globalización social, cultural y económica 
que produce - paradójicamente- una tendencia creciente hacia la concentración de sus efectos en el nivel local (Borja, 1994)" (Carrión, 2001, p. 9); y el desarrollo tecnológico, a través de la reducción de las distancias o la transformación de las mismas, y por los nuevos medios de comunicación como manera de socialización de la población.

\section{Cuenca, de ciudad compacta a ciudad dispersa}

Cuenca fue fundada oficialmente el 12 de abril de 1557 con el nombre de Santa Ana de los Ríos de Cuenca. Al igual que en la mayoría de ciudades latinoamericanas, se siguieron las Leyes de Indias, que implican un trazado en damero y manzanas de 84 metros de lado en torno a la plaza central (Figura 1). Desde alrededor de 1850, la economía de la ciudad se dinamizó por la exportación y comercialización del sombrero de paja toquilla, lo cual hizo que la ciudad experimentara un fuerte crecimiento hacia los cuatro costados del núcleo urbano (Carpio, 1979; Jaramillo, 1982) (Figura 1), principalmente por las vías de salida hacia distintas regiones del país: San Sebastián como salida a Molleturo y Guayaquil; San Roque como salida hacia Ońa y Loja; y El Vecino como salida hacia la capital, Quito (Carpio, 1979; Jaramillo, 1982) (Figura 2). Carpio (1979) cita a Estrella (1975), quien señala que en 1862 los sombreros de paja toquilla eran el segundo producto de exportación del Ecuador. Carpio agrega que la producción de sombreros fue la principal actividad económica de la provincia del Azuay hasta 1950. Este fenómeno va de la mano con lo que ocurría a nivel nacional a finales del siglo xix. Carrión cita a Guerrero (1980, p. 13) al respecto, quien señala: "La guerra civil de 1895 sella el proceso de unificación y constitución del Estado Nacional y marca, creemos, el comienzo de la dominación del modo de producción capitalista en el conjunto de la formación social ecuatoriana” (Carrión, 2010, p. 62).

Figura I | Mapa de Cuenca fundacional (1557) y mapa del crecimiento de Cuenca (1878)

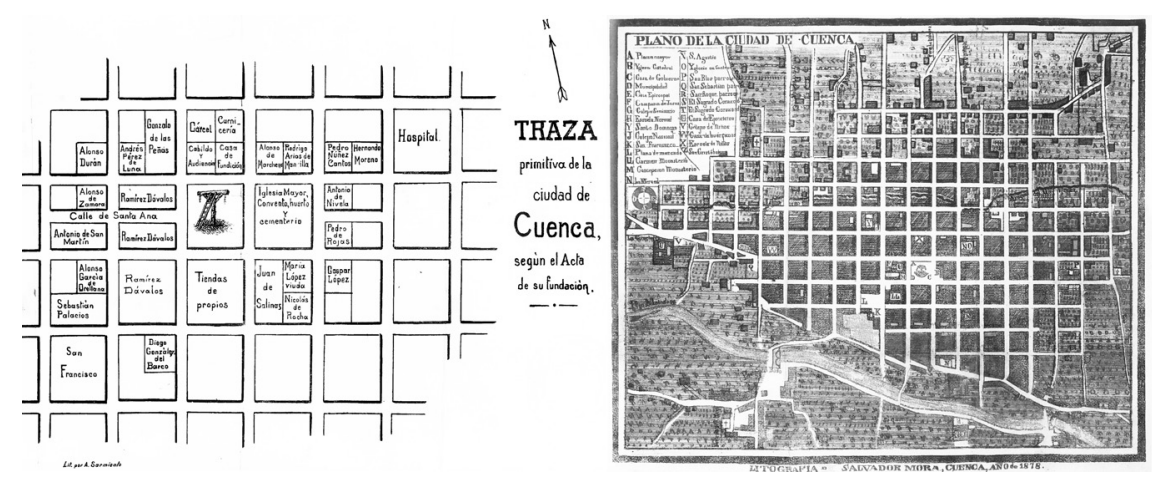

FUENTE I. MUNICIPALIDAD DE CUENCA (2008), PP. 74 Y IO7 
FIGURA 2 | Mapa del Ecuador entre 1906 y 1911

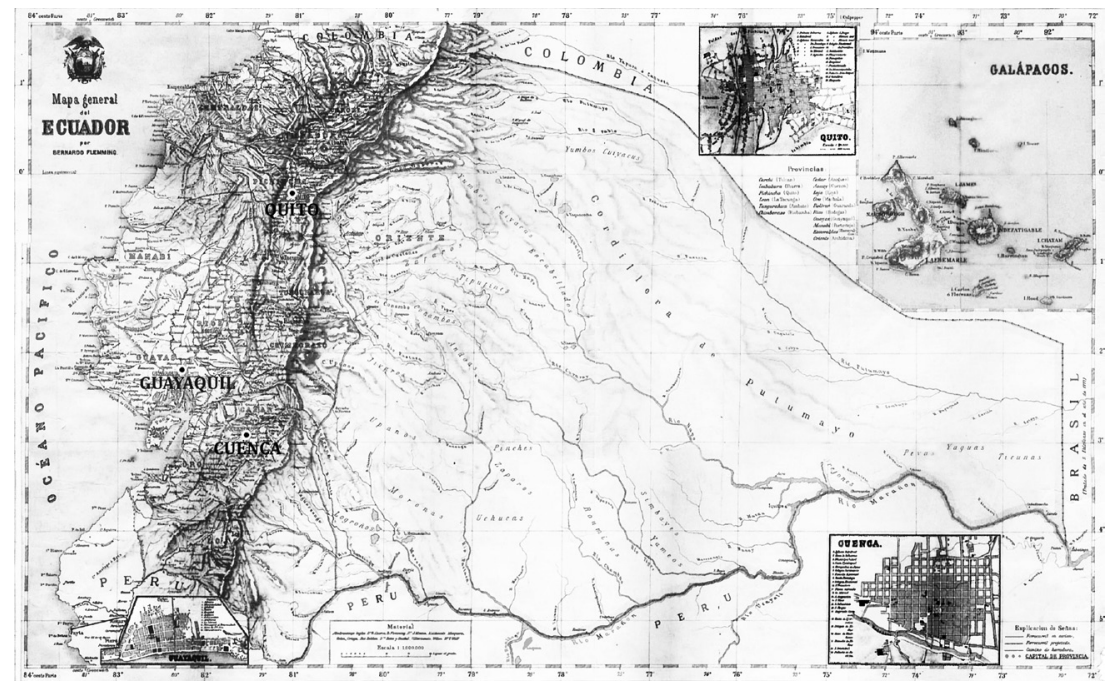

FUENTE I. MUNICIPALIDAD DE CUENCA (2008), P. I I 8

Ya en el siglo xx, en las décadas de los treinta y cuarenta, el "municipio" ecuatoriano se constituyó en el marco institucional para las vertientes tanto higienistas como planificadoras, por lo que se convirtió en el responsable del manejo del ambiente urbano (Carrión, 1998). En este contexto, a finales de los años cuarenta, el arquitecto uruguayo Gilberto Gatto Sobral elaboró el Anteproyecto del Primer Plan Regulador para Cuenca, con una visión a cincuenta ańos (Figura 3). En él plantea los principios de la zonificación, la racionalización, la higienización y la valoración económica, dividiendo la ciudad en zonas: comercial, industrial, cinturón agrícola y cinco zonas residenciales. Sin embargo, esta planificación, de acuerdo con Carpio (1979), no se pudo aplicar íntegramente, entre otras razones, debido a que la zonificación y las otras medidas propuestas eran teóricas: "Las zonas residenciales se disponían en forma complicada y presuponían una estructura social propia de un país industrial (...) en otro orden de cosas, no se pudo controlar el inevitable juego de los intereses particulares" (p. 69). Tal sería la razón, según el mismo Carpio, de que hasta la década de 1950 Cuenca mantuviera su organización urbana colonial.

Según Carrión (1998), a partir de los sesenta se generaliza el fenómeno urbano en el Ecuador y la población comienza a concentrarse en las ciudades, lo cual "ha convertido al Ecuador en un país de ciudades con población predominantemente urbana” (p. 12). Esta aseveración coincide con lo que sucede en Cuenca. Entre los años cincuenta y sesenta, la exportación del sombrero de paja toquilla decae y a partir de esta fecha comienza un proceso incipiente de industrialización. "En el siglo XIX adquiere significación extrarregional -y gran importancia económica local- la producción de sombreros de paja toquilla (...). Solo a partir de 1960 se instalan algunas fábricas cuya producción se comercializa a todo el país" (Carpio, 1979, p. 123). La fuerte migración campo-ciudad que provoca este cambio de modelo productivo 
implica modificaciones en materia de movilidad diaria y la conformación de nuevos asentamientos urbanos periféricos, como El Vecino y Totoracocha (Figura 4).

Figura 3 | Mapa del Plan Regulador de la ciudad de Cuenca, 1949. Elaborado por Gilberto Gatto Sobral

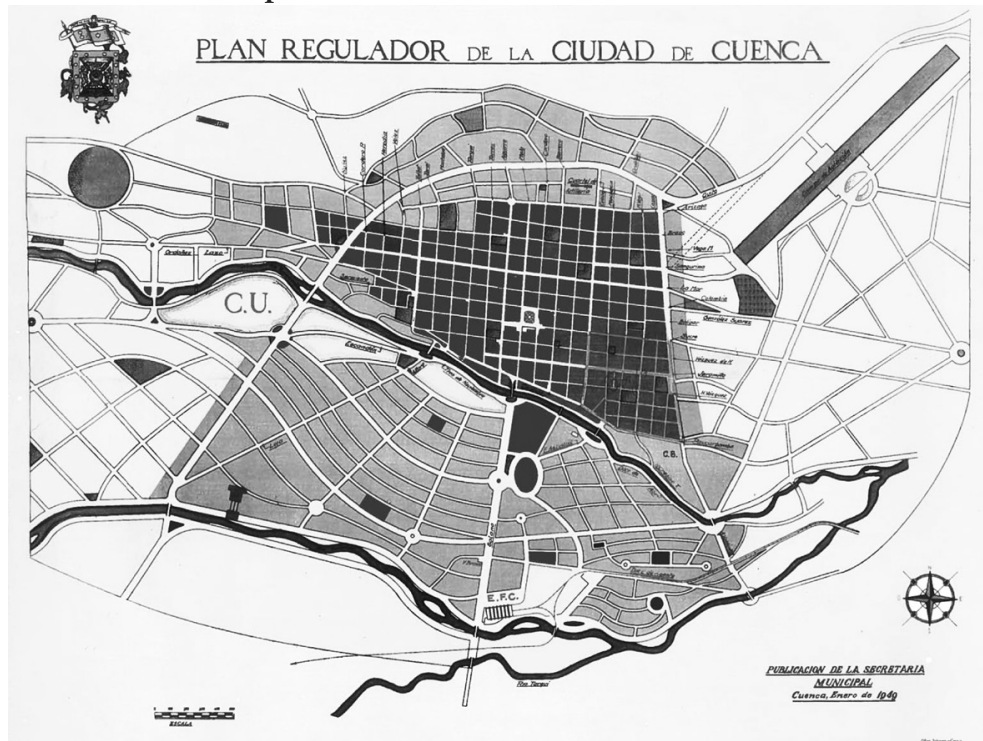

FUENTE I. MUNICIPALIDAD DE CUENCA (2008), P. I 57

FIgura 4 | La ciudad de Cuenca. Fotografía aérea de 1973

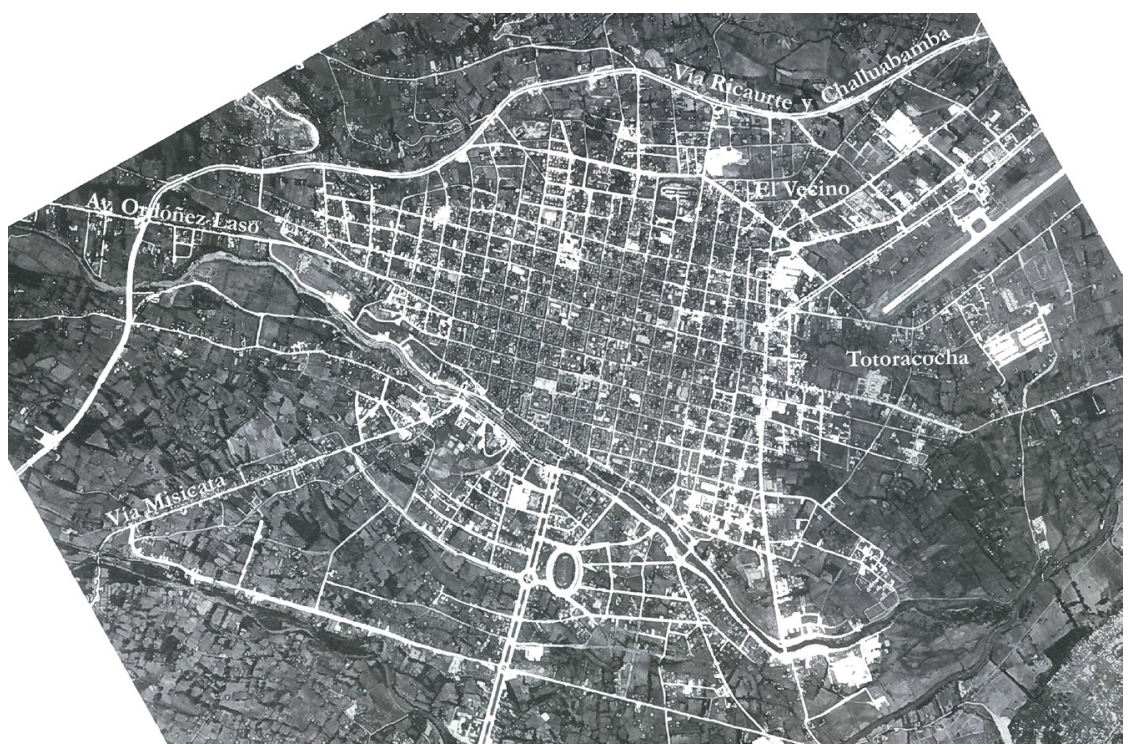

FUENTE I. MUNICIPALIDAD DE CUENCA (2008), P. 209 
El modelo de industrialización sustitutiva de importaciones, sumado al incremento de la capacidad económica del país como resultado de los ingresos provenientes del petróleo, activó al sector de la construcción y al sistema financiero comercial. En este contexto, se elaboró en 1971 el Plan Director de Desarrollo Urbano de la Ciudad de Cuenca y, más de una década después, en 1982, el Plan de Desarrollo Urbano del Área Metropolitana de Cuenca. Este último tuvo como finalidad descentralizar el centro histórico, reforzar nuevos centros urbanos, ocupar el suelo vacante en las zonas consolidadas, proteger los márgenes de los ríos, y controlar el crecimiento sobre los corredores y suelos agrícolas (Jaramillo, 1982). Posteriormente, en la década de los noventa, con el auge de las teorías del desarrollo sustentable a nivel mundial - priorizado en el caso de Latinoamérica por la Conferencia en Río de Janeiro de las Naciones Unidas sobre el Medio Ambiente y el Desarrollo, en la cual se aprobó la Agenda 21-, se evidencia la necesidad de controlar el crecimiento urbano. Es así que en 1998, el Municipio de Cuenca emitió la Ordenanza que Sanciona el Plan de Ordenamiento Territorial del Cantón, la cual establece el límite urbano, así como las normas para la infraestructura y la construcción. Esta Ordenanza fue reformada, actualizada y codificada en el año 2003. Tras esta reforma a nivel de cantón, fueron varios los planes parciales elaborados en las zonas hacia donde existe presión inmobiliaria; es el caso, por ejemplo, del Plan Parcial de Challuabamba aprobado en mayo de 2008 (Figura 5).

\section{Figura 5 | Mapa del límite urbano actual de la ciudad de Cuenca}

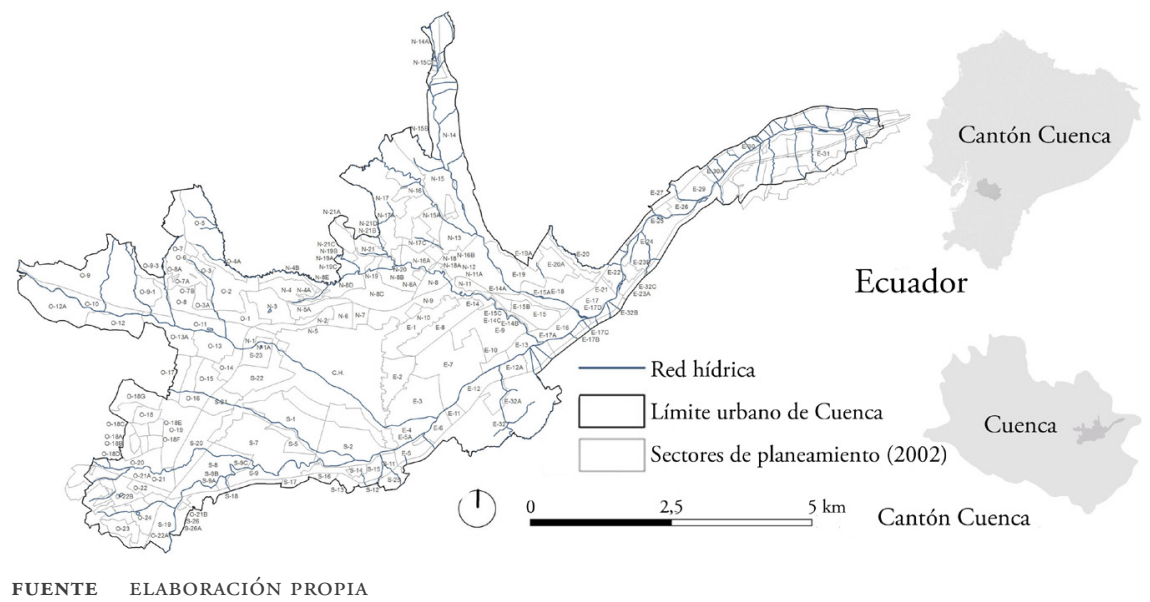

A nivel nacional se puede decir que, a partir de la asamblea constituyente, en el año 2008, la planificación da un giro. La nueva Constitución y los posteriores Códigos Orgánicos de Organización Territorial, Autonomía y Descentralización y de Planificación y Finanzas Públicas, y el Plan Nacional del Buen Vivir, inciden en la planificación de los municipios. A diferencia de la Constitución anterior, del ańo 1998, en donde se abre el país al libre mercado y a la globalización, la actual Carta Magna pretende una inserción estratégica en el mundo, pero tiene como objetivo 
principal la consecución del buen vivir ${ }^{1}$ a través de la redistribución de la riqueza, y de propiciar la sustentabilidad social y ambiental, y la descentralización: "A diferencia de la anterior, la Constitución aprobada en 2008 recupera a la planificación y le otorga un papel decisivo en relación a temas directamente involucrados con el desarrollo humano en el Ecuador, tales son los principios contemplados en ella, el ejercicio de los derechos y los denominados 'regímenes de desarrollo y del buen vivir"” (Pauta, 2013, p. 57).

La primera competencia otorgada a los municipios en la actual Constitución es "1. Planificar el desarrollo cantonal y formular los correspondientes planes de ordenamiento territorial, de manera articulada con la planificación nacional, regional, provincial y parroquial, con el fin de regular el uso y la ocupación del suelo urbano y rural" (Constitución del Ecuador, 2008). Es así que en enero de 2012 se emitió la Ordenanza de Aprobación del Plan de Desarrollo y Ordenamiento Territorial (РDOT) para Cuenca, cuyo alcance es de nivel cantonal y parte de una visión integral de desarrollo, y no tiene por objetivo llegar al detalle de la normativa urbana (Plan de Desarrollo y Ordenamiento Territorial del Cantón Cuenca, 2011). El Plan de Ordenamiento Urbano será un producto posterior al РDOT, que se aprobó en primer debate el 11 de febrero de 2015 (http://www.cuenca.gob.ec).

\section{Cuenca, estudio de densidad urbana desde 1950}

El cantón Cuenca constituye el tercero del Ecuador en población, con 505.585 habitantes según el censo 2010 (www.inec.gob.ec), de los cuales 331.885 habitan en el área urbana, cuya extensión es de 7.248 hectáreas. Sin embargo, indica Pauta (2013), lo que el Instituto Nacional de Estadísticas y Censos (INEC) ha considerado como "población urbana" no es un reflejo de la realidad, ya que se toma exclusivamente a la residente en las cabeceras cantonales, y como rural a la "población de todas las cabeceras parroquiales, independientemente de su tamaño y de las características de estas unidades espaciales" (p. 43). Y ello cuando, como en el caso de Cuenca, existen cabeceras parroquiales cuya "población muestra una estructura ocupacional vinculada a la base económica urbana contigua o cercana a más de que depende en forma más o menos importante de los bienes y servicios que ella produce" (Pauta, 2013, p. 42). No obstante, para efectos de este artículo y por ser la fuente disponible, se tomarán los datos de "población urbana" de la cabecera cantonal de Cuenca, obtenidos en el vir Censo Nacional de Población y vi de Vivienda de 2010 .

La densidad constituye una variable fundamental de la compacidad de una ciudad. Entendemos por "compacidad" la combinación de la densidad urbana, el tipo de ocupación, el reparto del viario público, la proximidad a redes de transporte alternativo al automóvil, la accesibilidad peatonal, el porcentaje de condominio

1 La Constitución del Ecuador señala, en su Preámbulo: “Decidimos construir: Una nueva forma de convivencia ciudadana, en diversidad y armonía con la naturaleza, para alcanzar el buen vivir, el sumak kawsay; una sociedad que respeta, en todas sus dimensiones, la dignidad de las personas y las colectividades; un país democrático, comprometido con la integración latinoamericana -sueńo de Bolívar y Alfaro-, la paz y la solidaridad con todos los pueblos de la tierra...”. 
cerrado y de espacio libre. La densidad, pues, puede ser medida por el número de viviendas y/o de habitantes en un área determinada.

El análisis en la ciudad de Cuenca se hace a partir de 1950, ya que, tal como se menciona anteriormente, es desde mediados del siglo anterior que la ciudad inicia su proceso de expansión fuera del casco histórico, y es a contar de dicho año que se dispone de datos confiables de población: "Es difícil tener una visión más o menos precisa del crecimiento demográfico de Cuenca antes del 1950. Los datos anteriores al siglo XVIII, solo son apreciaciones de muy discutible exactitud. El estudio histórico demográfico de Hamerly aporta datos muy útiles acerca de la población del Distrito de Cuenca, a finales del siglo xvin y comienzos del xix (...). Pero, nuevamente, caben muchas dudas respecto a los cálculos hechos en el resto del siglo xIX y la primera mitad del xx" (Carpio, 1979, p. 101).

\section{CUADro I $\mid$ Densidad bruta de la ciudad de Cuenca a partir de 1950}

\begin{tabular}{|c|c|c|l|l|l|c|}
\hline \multicolumn{6}{|c|}{ ANÁLISIS DE DENSIDAD URBANA EN LA CIUDAD DE CUENCA I950 - 2OIO } \\
\hline $\begin{array}{c}\text { AÑO DE } \\
\text { CENSO }\end{array}$ & $\begin{array}{c}\text { POBLACIÓN } \\
\text { HAB }\end{array}$ & $\begin{array}{c}\text { AÑO DE } \\
\text { LÍMITE } \\
\text { URBANO }\end{array}$ & $\begin{array}{c}\text { FUENTE } \\
\text { DEL LÍMITE } \\
\text { URBANO }\end{array}$ & $\begin{array}{c}\text { ÁREA BRUTA } \\
\text { HA }\end{array}$ & $\begin{array}{c}\text { PORCENTAJE } \\
\text { DE ÁREA } \\
\text { LIBRE \% }\end{array}$ & $\begin{array}{c}\text { DENSIDAD } \\
\text { BRUTA HAB/ } \\
\text { HA }\end{array}$ \\
\hline 1950 & 39.983 & 1946 & municipal & 288,29 & 32,89 & 138,69 \\
\hline 1962 & 60.402 & 1968 & municipal & $2.237,30$ & 39,4 & 27 \\
\hline 1974 & 104.470 & 1974 & censal & 2.317 & 41,64 & 45,09 \\
\hline 1982 & 152.406 & 1982 & censal & 2674,99 & 41,42 & 56,97 \\
\hline 1990 & 194.981 & 1990 & censal & $4.580,21$ & 43,43 & 42,57 \\
\hline 2001 & 277.374 & 2001 & censal & 6395,99 & 45,8 & 43,37 \\
\hline 2010 & 329.928 & 2010 & censal & $7.248,23$ & 46,2 & 45,52 \\
\hline
\end{tabular}

FUENTE ELABORACIÓN PROPIA

Hacia 1950, la zona urbana de Cuenca contaba, según el Primer Censo Nacional de Población, con 39.983 habitantes; y según datos municipales, del año 1946, con un área urbana de 288,29 hectáreas, de lo cual se infiere una densidad bruta de 138,69 hab/ha. Espacialmente la ciudad estaba constituida por la malla en forma de damero con solo 13 manzanas en sentido norte-sur y 23 en sentido este-oeste. Los espacios públicos y libres se encontraban bien distribuidos, es decir, a dos o tres manzanas de distancia entre ellos. La población podía vivir, trabajar, desplazarse y acceder a espacios de ocio sin dificultad. Hay que tener en cuenta que la presencia del automóvil en aquel entonces no era representativa, por lo que la ciudad mantenía su organización espacial compacta.

Con posterioridad a esta fecha empieza, en parte, la implantación de la nueva planificación de la ciudad elaborada por el arquitecto Gilberto Gatto Sobral en el Primer Plan Regulador. Es por ello que, para el censo de 1962, Cuenca poseía 60.402 habitantes, un poco menos del doble que en 1950, mientras que, según datos municipales de 1968, el área urbana se había incrementado siete veces a 2.237,30 hectáreas, por lo que, en los ańos sesenta, se tiene una densidad bruta de 27 hab/ ha. Esta nueva ocupación de las áreas periféricas provocó una disminución de la densidad en cinco veces su valor con respecto a los datos de 1950, lo que corrobora 
lo señalado por Carrión (1994), quien manifiesta, con respecto al crecimiento de las ciudades ecuatorianas, que "esta modalidad de crecimiento se realiza sin un requisito real o, lo que es lo mismo, es una expansión urbana de los límites de la ciudad por encima de las demandas de la población. Esto se explica por el hecho de que la población crece a un ritmo inferior de lo que ocurre con la mancha urbana, al grado que las densidades de las ciudades bajan” (p. 78). Tal como se mencionó anteriormente, la planificación de Gatto Sobral responde a criterios del Congreso Internacional de Arquitectura Moderna (CIAM); por ende, da prioridad al automóvil y sectoriza la ciudad, incrementando las distancias entre vivienda y trabajo. Esto último intensifica las inequidades y obliga a una dependencia del transporte público para un sector de la ciudad y a la dependencia del automóvil para otro.

Los censos de 1974 y 1982, por otro lado, evidencian una consolidación del área urbana. En 1974, la densidad bruta es de 45,09 hab/ha, mientras que en 1982 es de 56,97 hab/ha; como se constata, la densidad se incrementa, aproximadamente, en doce puntos entre censos. Se consolida la ocupación hacia la zona de El Vecino y Totoracocha por la activación del sector de la construcción, que respondía al modelo económico imperante y al boom petrolero (Figura 6).

FIgura 6 | Densidades brutas históricas de la ciudad de Cuenca desde 1946 hasta 1982

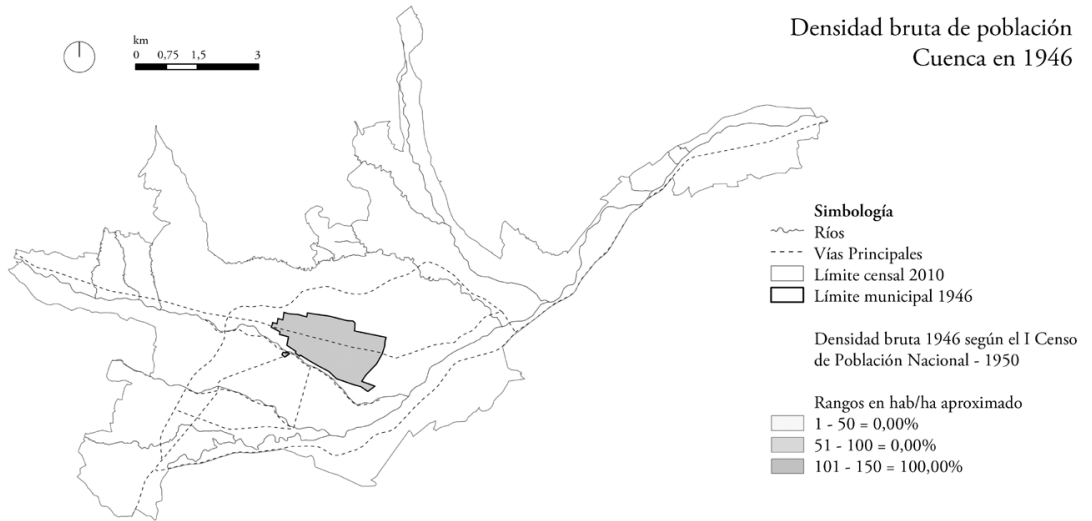

Densidad bruta de población Cuenca en 1968

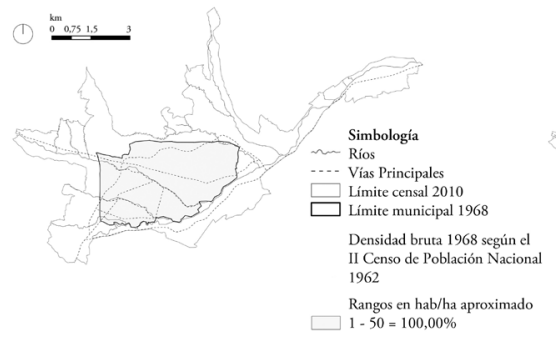

FUENTE ELABORACIÓN PROPIA
Densidad bruta de población Cuenca en 1982

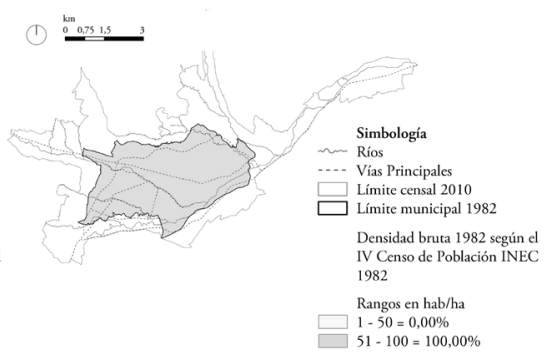


Entre el censo de 1982 y el de 1990, nuevamente se observa un decrecimiento importante en la densidad. Esto se debe a que la población se incrementa, en esos ocho ańos, en un 28\%, mientras que el área urbana crece aproximadamente en un $71 \%$, por la ocupación de los corredores Ordóñez Laso, vía a Misicata, vía a Ricaurte y Challuabamba, entre otros. Este fenómeno ocurre a pesar de que el Plan de Desarrollo Urbano del Área Metropolitana de Cuenca de 1982 propone, de manera teórica, controlar el crecimiento sobre los corredores y promueve la ocupación del suelo vacante dentro del área consolidada, lo cual demuestra que hacen falta acciones prácticas que motiven el cumplimiento del Plan y que frenen el crecimiento desmedido. Los censos de 1990, 2001 y 2010 evidencian una relativa estabilidad en cuanto a las densidades (Figura 7). Cabe anotar que las áreas utilizadas para el cálculo de las densidades corresponden a las dadas por el límite censal; sin embargo, para el análisis de los años 1950 y 1962 en este estudio, se toman los límites urbanos de la cartografía municipal del año 1946 y 1968, respectivamente, por carecer de cartografía censal.

FIgURA 7 Densidades brutas históricas de la ciudad de Cuenca desde 1990 hasta 2010

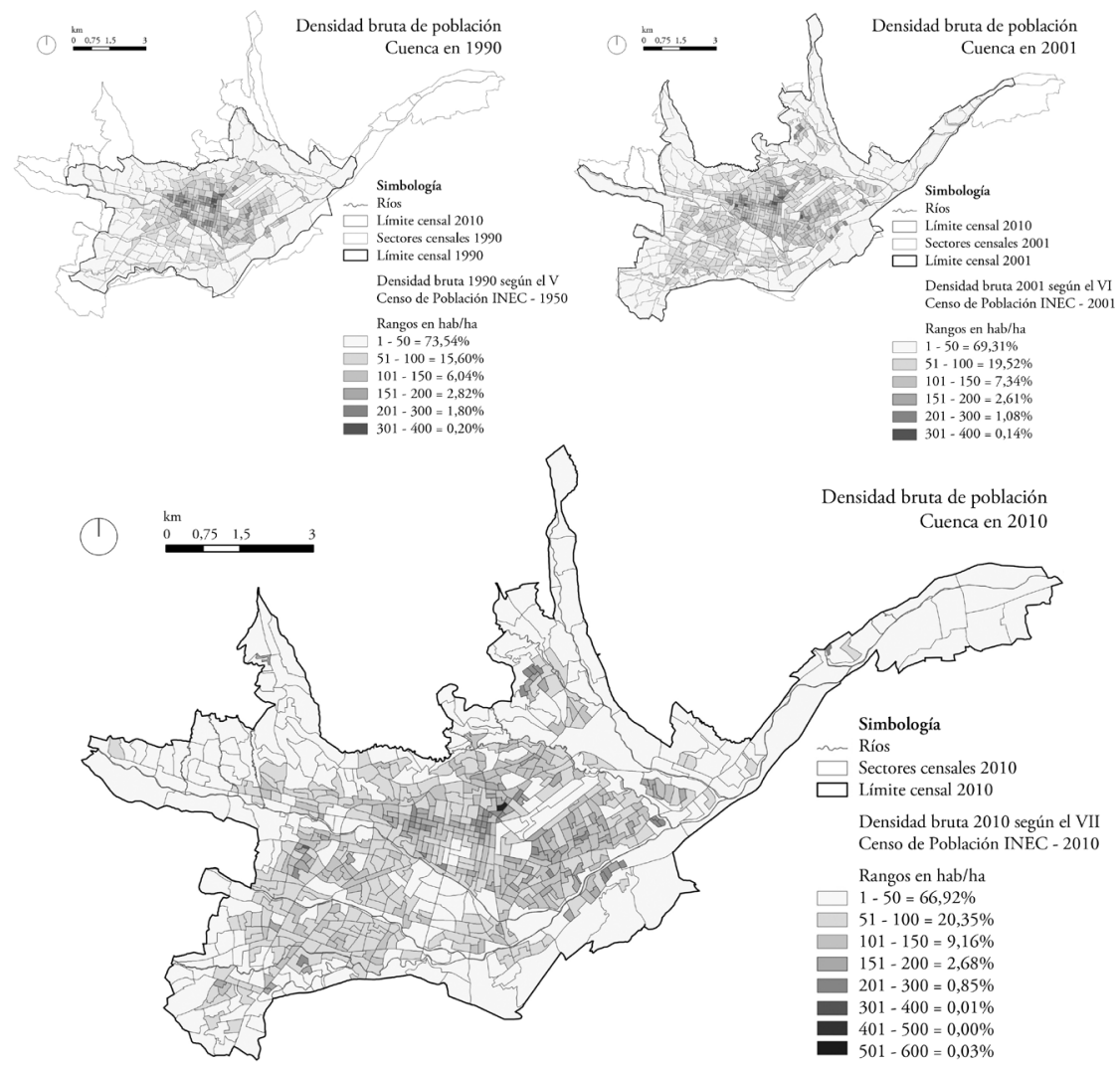

FUENTE ELABORACIÓN PROPIA 
A través de una proyección tendencial de la población de la ciudad de Cuenca en que se consideran dos variables -crecimiento natural y migración-, el escenario más probable de crecimiento de la población en la zona urbana de Cuenca nos muestra una población para el año 2055 de 861.682, en relación con los 329.928 habitantes que existen en 2010. Es importante anotar que para estos valores se considera el saldo migratorio implícito. Si, hipotéticamente, el perímetro urbano se mantuviera igual al actual -es decir, si Cuenca continuara con un área urbana de 7.248 hectáreas hasta el año 2055-, su densidad bruta sería de 118,88 hab/ha (Figura 8 y Cuadro 2).

FIGURA 8 | Proyección tendencial de la población de la ciudad de Cuenca

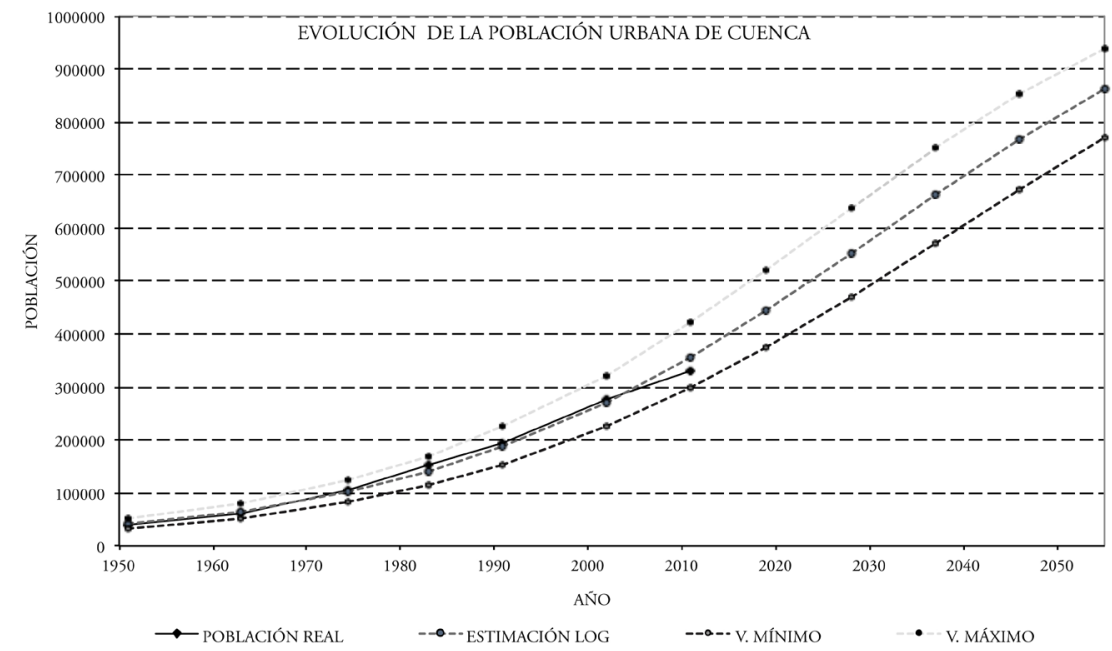

FUENTE ELABORACIÓN PROPIA

CUADro 2 Proyección tendencial de la población de la ciudad de Cuenca

\begin{tabular}{|c|l|c|}
\hline \multicolumn{1}{|c|}{ AÑo } & \multicolumn{1}{|c|}{ HIPÓTESIS } & POBLACIÓN TOTAL \\
\hline \multirow{3}{*}{2019 (corto plazo) } & MAX PRO* & 520.297 \\
\cline { 2 - 3 } & INTERMEDIO (abierta)** & 393.408 \\
\cline { 2 - 3 } & MED PRO* & 444.810 \\
\cline { 2 - 3 } & INTERMEDIO (semisuma) & 409.440 \\
\cline { 2 - 3 } & MIN PRO* & 374.069 \\
\hline \multirow{3}{*}{2055 (largo plazo) } & MAX PRO* & 939.090 \\
\cline { 2 - 3 } & INTERMEDIO (semisuma) & 900.386 \\
\cline { 2 - 3 } & MED PRO* & 861.682 \\
\cline { 2 - 3 } & INTERMEDIO (semisuma) & 815.985 \\
\cline { 2 - 3 } & MIN PRO* & 770.288 \\
\hline
\end{tabular}

* Valores de R2=0,993 I, índice más confiable, Que corresponde a "Regresión logistica” I950-20IO

** VALORES SEGÚN EL MÉTODO DE “COHORTES TF 200I-20IO"

FUENTE ELABORACIÓN PROPIA 
La ordenanza actualmente en vigencia, del año 2003, propone, de manera teórica, la densificación de los distintos sectores de planeamiento. Sin embargo, la diferencia entre los valores de densidad propuestos en dicha ordenanza y la densidad de viviendas real es muy grande. Luego de realizar un análisis comparativo entre estos valores, comprobamos que en el $97,27 \%$ de los casos las densidades reales son menores a las autorizadas, lo cual evidencia una tendencia hacia la construcción en baja altura y hacia las bajas densidades (Cuadro 3).

CuAdro 3 | Diferencia de Densidad Neta de Vivienda (DNV) entre la Ordenanza para Sectores de Planeamiento 2003 y el vi Censo de Población 2010

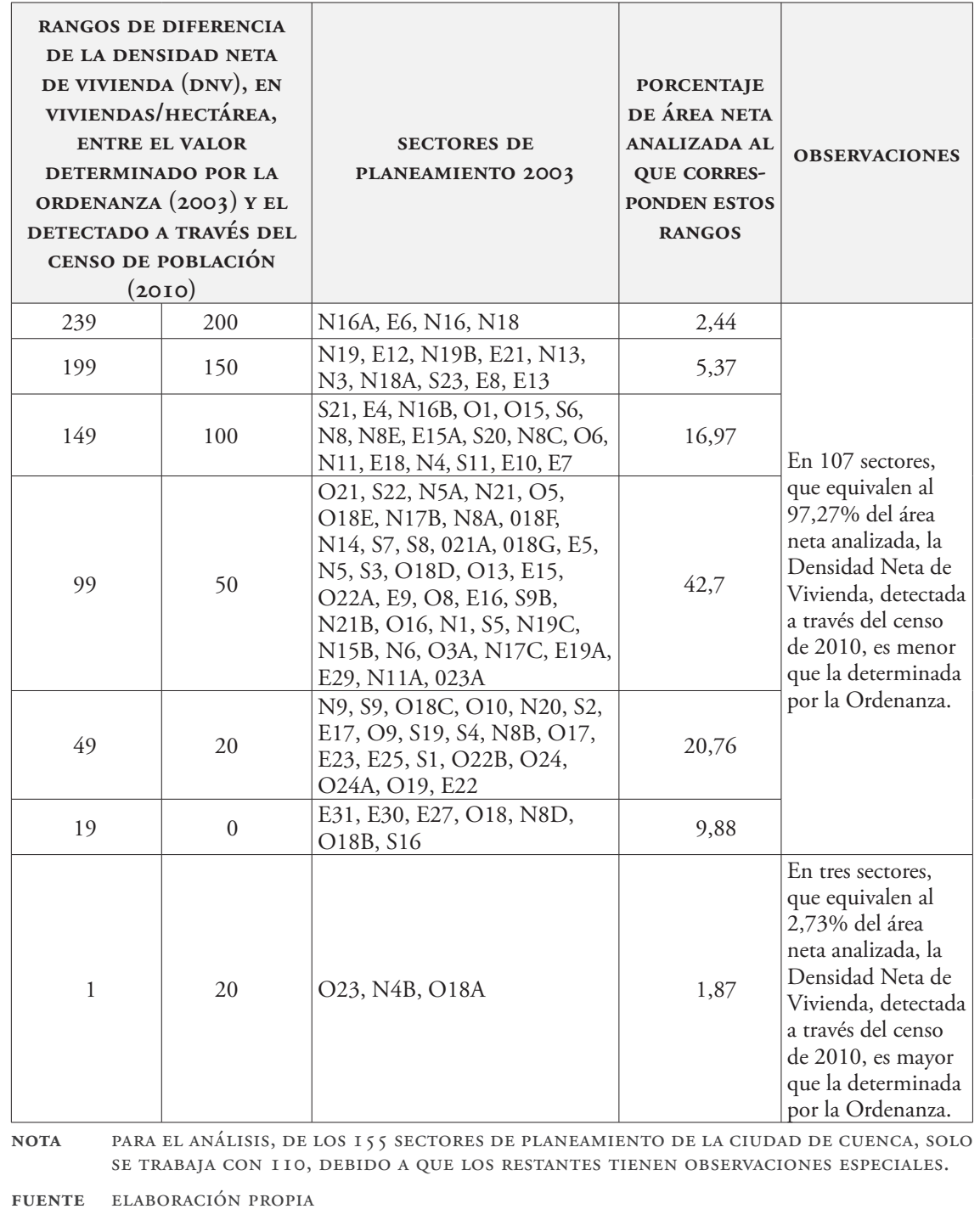




\section{Conclusiones y recomendaciones}

Una manera de frenar los impactos negativos, tanto ambientales como económicos y sociales, de la ciudad dispersa es mediante la densificación planificada y en relación con todas las variables de una ciudad habitable, que considere la calidad de vida de las personas como el eje de la reflexión. El artículo analiza la variación de las densidades de la ciudad de Cuenca con el fin de demostrar la tendencia innecesaria de crecimiento disperso y poco planificado. El fin último de la investigación es motivar la búsqueda de estrategias de planificación y diseño urbano para aprovechar de mejor manera el suelo dentro de la ciudad construida y evitar así los múltiples inconvenientes de la dispersión. Sin duda, entre las estrategias para alcanzar la sustentabilidad de la ciudad se encuentra la densificación, particularmente en el caso de Cuenca, que en su momento fue una ciudad compacta y habitable. Y consideramos que podría volver a serlo si se aplican las medidas correctivas necesarias. Este aspecto es solo uno más de los necesarios para construir un modelo de ciudad habitable y sustentable propio.

Cuenca, al ser una ciudad intermedia, no presenta todavía, con la misma intensidad, los efectos negativos sociales y ambientales de un modelo disperso; sin embargo, las tendencias nos ponen en alerta sobre lo que podría suceder si no se da un giro en el modelo de ciudad que queremos construir. Hemos observado que la ordenanza municipal vigente promueve la densificación, pero únicamente de manera teórica, ya que no contempla una verdadera política de incentivos para ello, ni trata sobre los aspectos colaterales que se deben tomar en cuenta cuando se propone densificar. La densificación tiene que ir de la mano con estrategias de movilidad, espacio público, complejidad urbana, entre otras. Los incentivos a la densificación deberían promover un uso adecuado de lotes vacantes, que en la ciudad de Cuenca representan el 10,5\% (I. Municipalidad de Cuenca, 2014), valor suficientemente alto como para permitir la aplicación de modelos de densificación adecuados.

$\mathrm{Al}$ profundizar sobre la ordenanza vigente, se observa que ella responde a un modelo de ciudad enfocada al automóvil, que en ningún caso permitiría un modelo de ciudad compacta que priorice al ciudadano de a pie. Existe un sinnúmero de artículos de la Ordenanza que promueven, defienden y legalizan el uso del automóvil, en detrimento de otros sistemas de movilidad. Este es, sin duda, un tema de investigación que deberá desarrollarse y profundizarse con urgencia si queremos que el nuevo modelo de ciudad se haga realidad.

Vale la pena subrayar el hecho de que, si la tendencia de crecimiento poblacional se mantiene en los próximos cuarenta ańos, Cuenca podría mantener su límite urbano sin necesidad de llegar a altas densidades. Cuando se habla de valores óptimos de densificación en otras ciudades del mundo, se recomienda 60 viv/ha (Rueda, 2008); es decir, valores aproximados de 240 hab/ha. Cuenca no requeriría valores tan altos, pues para acoger a la población proyectada al 2055 (Figura 8 y Cuadro 2) en la misma área urbana, la densidad bruta no sobrepasaría los 118,88 hab/ha. Entre las medidas para lograr este objetivo estaría la ocupación de los lotes vacíos a través de modelos específicos de densificación acordes con las distintas tipologías morfológicas de la ciudad, y a través de políticas de densificación desde 
una perspectiva de sustentabilidad que definiera el uso del espacio público para la población y no solo para el automóvil.

Existen dos escenarios posibles: continuar con el actual ritmo de crecimiento, dispersión y bajas densidades, que podrían, hipotéticamente, implicar que hasta el año 2055 el área urbana de Cuenca crezca a 19.369 ha frente a las 7.248 ha que ocupa actualmente; o plantearnos un escenario de densificación sustentable dentro de un modelo de ciudad habitable enfocado al respeto al ser humano y al medioambiente.

Sin embargo, y a pesar de estas buenas intenciones, no podemos olvidar que la producción de ciudad no es solo un acto de voluntad que obtenga de la academia las posibles soluciones científicas y teóricas. En su construcción aparecen desafíos económicos, fiscales, ambientales, institucionales y de gobernabilidad que se relacionan, íntimamente, con las diferentes áreas y niveles de gobierno que deben coordinarse entre sí, y en donde, además, se tienen que articular el sector privado y la sociedad civil. La voluntad política, el equilibrio en la lucha de intereses, el mercado y la participación ciudadana en la toma de decisiones constituyen componentes fundamentales para que las iniciativas en favor de una ciudad más humana se hagan realidad.

\section{Referencias bibliográficas}

Arbury, J. (2005). From urban sprawl to compact city. An analysis of urban growth management in Auckland. Tesis de maestría, Auckland University, Auckland. Disponible en http:// transportblog.co.nz/wp-content/uploads/2009/06/thesis.pdf

Barton, H., Melia, S. \& Parkhurst, G. (2011). The paradox of intensification, Journal of Transport Policy, 18(1), 46-52. Disponible en http://dx.doi.org/10.1016/j.tranpol.2010.05.007

Borsdorf, A. (2003). Cómo modelar el desarrollo y la dinámica de la ciudad latinoamericana. EURE, 29(86), 37-49. Disponible en file:///Users/Usuario1/Downloads/1265-59881-SM.pdf

Carpio, J. (1979). Cuenca: su geografía urbana. Cuenca: López Monsalve.

Carrión, F. (1994). En busca de la ciudad perdida. Quito: CODEL.

Carrión, F. (1998). Espacio urbano: hacia la ciudad sustentable. Seminario Ciudades Sustentables (pp. 1-37). Cuenca. No publicado.

Carrión, F. (2001). Las nuevas tendencias de la urbanización en América Latina. En F. Carrión (Ed.), La ciudad construida. Urbanismo en América Latina (pp. 7-24). Quito: FLACsoEcuador.

Carrión, F. (2010). Ciudad, memoria y proyecto. Quito: OLACCHI-Municipio Metropolitano de Quito.

Cervero, R. (1998). The transit metropolis. Washington DC: Island Press.

Comisión Económica para América Latina y el Caribe (CEPAL). (1998). Ciudades intermedias de América Latina y el Caribe: Propuestas para la gestión urbana. Compilado por R. Jordán $\&$ D. Simioni. Santiago: Autor. Disponible en http://repositorio.cepal.org/bitstream/ handle/11362/31024/S9800066_es.pdf?sequence=1 
Constitución Política del Ecuador. (2008). Disponible en http://www.asambleanacional.gov. ec/documentos/constitucion_de_bolsillo.pdf

De Mattos, C.A. (2010). Globalización y metamorfosis metropolitana en América Latina. De la ciudad a lo urbano generalizado. Revista de geografia Norte Grande, (47), 81-104. Disponible en http://dx.doi.org/10.4067/S0718-34022010000300005

Dimitriou, H. (2011). Transport city development: Understanding the fundamentals. En H. Dimitriou \& R. Gakenheimer (Eds.), Urban transport in the developing world. A handbook of policy and practice (pp. 8-39). Cheltenham: Edward Elgar.

Duany, A., Plater-Zyberk, E. \& Speck, J. (2000). Suburban nation: The rise of sprawl and the decline of the American dream. Nueva York: North Point Press. Disponible en http:// transportblog.co.nz/wp-content/uploads/2009/06/thesis.pdf

Estrella, P. (1975). La Fundación de la Universidad de Cuenca y su vinculación con la realidad socio-económica de la época. En Revista del Instituto de la Investigación Regional de la Universidad de Cuenca. Cuenca: Mimeo.

Ewing, R., Meakins, G., Bjarnson, G. \& Hilton, H. (2011). Transportation and land use. En A. Dannenberg (Ed.), Making healthy places: designing and building for health, wellbeing and sustainability (pp. 149-169). Washington, DC: Island Press.

Gakenheimer, R. (2011). Land use and transport in rapidly motorizing cities: contexts of controversy. En H. Dimitriou \& R. Gakenheimer (Eds.), Urban transport in the developing world. A handbook of policy and practice (pp. 40-68). Cheltenham: Edward Elgar.

Guerrero, A. (1980). Los oligarcas del Cacao. Quito: El Conejo.

Herce, M. \& Magrinyá, F. (2010). La ingeniería en la evolución urbanistica (2a ed.). Barcelona: Universidad Politécnica de Cataluña.

I. Municipalidad de Cuenca. (2008). Planos e Imágenes de Cuenca. Cuenca: Autor.

I. Municipalidad de Cuenca. (2014). Estudio para el Plan de Ordenamiento Urbano de Cuenca. Cuenca: Autor.

Jaramillo, C. (1982). Evolución histórica de la ciudad de Cuenca. Revista del Instituto Geográfico Militar, (26), 117-137.

Lehmann, S. (2010). Green Urbanism: Formulating a Series of Holistic Principles. SAPIENS, 3(2), 1-10. Disponible en file:///Users/Usuario1/Downloads/sapiens-1057-3-2-greenurbanism-formulating-a-series-of-holistic-principles.pdf

Ludeña, W. (2006). Ciudad y patrones de asentamiento. Estructura urbana y tipologización para el caso de Lima. EURE, 32(95), 37-59. Disponible en http://www.eure.cl/index. php/eure/article/view/1292

Ministerio de Medio Ambiente y Medio Rural y Marino, España (Dirección General de Calidad y Evaluación Ambiental) \& Agencia de Ecología Urbana de Barcelona (2007/2009). Libro verde del medio ambiente urbano. Tomos I y II. [online]. Disponible en http:// www.bcnecologia.net/es/proyectos/libro-verde-de-medio-ambiente-urbano-tomo-i-yii

Moliní, F. \& Salgado, M. (2010). Superficie artificial y viviendas unifamiliares en España, dentro del debate entre ciudad compacta y dispersa. Boletín de la Asociación de Geógrafos Españoles (54), 125-147. Disponible en http://www.boletinage.com/ articulos/54/06\%20AGE\%2054.pdf 
Muñiz, I., Calatayud, D. \& García, M.A. (2010). Sprawl. Causas y efectos de la dispersión urbana. En F. Indovina, La ciudad de baja densidad. Lógicas, gestión y contención (pp. 307-347). Barcelona: Diputació de Barcelona.

Navarro, J. (2011). Aproximación a la génesis de la contribución de la densidad en la noción de "ciudad compacta”. EURE, 32(112), 23-41. Disponible en http://www.scielo.cl/scielo. php?pid=S0250-71612011000300002\&script=sci_arttext

Neuman, M. (2005). The compact city fallacy. Journal of Planning Education and Research, 25(1), 11-26. doi: 10.1177/0739456X04270466

Nozzi, D. (2003). Road to ruin: an introduction to sprawl and how to cure it. Westport, Conn: Praeger.

Pauta, F. (2013). Ordenación territorial y urbanistica: un camino para su aplicación en el Ecuador. Cuenca: Monsalve Moreno.

Rueda, S. (2008). Plan Especial de Indicadores de Sostenibilidad Ambiental de la Actividad Urbanistica de Sevilla. Gerencia de Urbanismo, Ayuntamiento de Sevilla. Disponible en http://scholar.google.com/scholar?hl=en\&btnG=Search\&q=intitle:Plan+Especial+de+I ndicadores+de+Sostenibilidad+Ambiental+de+la+Actividad+Urbanística+de+Sevilla\#0

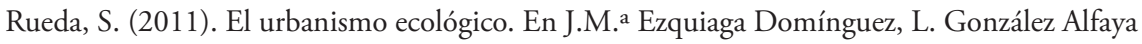
(Eds.), Transformaciones urbanas sostenibles (pp. 235-267). Madrid: Universidad Internacional Menéndez Pelayo.

Rufí J. \& Pinatella, M. (2007). Políticas para la contención del proceso de urbanización dispersa. En F. Ondavina (Ed.), La ciudad de baja densidad (pp. 351-379). Barcelona: S.A. de Litografía.

United Nations (1987). Report of the World Commission on Environment and Development: Our Common Future (Brundtland Report). Un Documents [online]. Disponible en http:// www.un-documents.net/our-common-future.pdf

Verdaguer, C. (2011). Modelos de desarrollo urbano y densidades edificatorias. El reciclaje de la ciudad en el ámbito español. Cambio global España 2020/50. Programa "Ciudades". Disponible en http://oa.upm.es/5844/1/1_CG2020_ProgramaCiudades_VERDAGUER_Modelosdedesarro.pdf 